\title{
Electroluminescence at Si band gap energy based on metal-oxide-silicon structures
}

\author{
Ching-Fuh Lin, ${ }^{\text {a) }}$ C. W. Liu, Miin-Jang Chen, ${ }^{\text {b) }}$ M. H. Lee, and I. C. Lin \\ Department of Electrical Engineering, National Taiwan University, Taipei, Taiwan, Republic of China
}

(Received 11 August 1999; accepted for publication 14 March 2000)

\begin{abstract}
Room-temperature electroluminescence corresponding to Si band gap energy from metal-oxidesemiconductor structures on both $p$-type and $n$-type $\mathrm{Si}$ is observed. With very thin oxide grown by rapid thermal oxidation, the metal-oxide-semiconductor structures behave like light emitting diodes. Luminescence is observed under forward bias even with a current density as low as 0.67 $\mathrm{A} / \mathrm{cm}^{2}$. The physical reason for the electroluminescence is discussed and attributed to the localized wave function that leads to the spread of momentum. As a result, the spread momentum causes the electron-hole radiative recombination to occur relatively easily. (c) 2000 American Institute of Physics. [S0021-8979(00)04612-0]
\end{abstract}

Silicon is the most important semiconductor material for the integrated-circuit industry. However, its indirect band gap makes it hardly emit light, leading to limited applications of silicon in areas of optics and optoelectronics. For decades, many efforts had been devoted to converting silicon to a light-emitting material or causing luminescence on silicon substrates. Those efforts include porous silicon-based devices, ${ }^{1-3}$ nanocrystalline $\mathrm{Si}^{4,5} \mathrm{Si}^{+}$-implanted $\mathrm{SiO}_{2},{ }^{6,7} \mathrm{Er}$ doped $\mathrm{Si},{ }^{8,9}$ deposition of polymer on $\mathrm{Si}$ substrates, ${ }^{10}$ growth of $\mathrm{GaN}$ on Si substrates, ${ }^{11,12}$ and so on. Recently, visible electroluminescence had also been reported from an $\mathrm{Ag} / \mathrm{SiO}_{2} / \mathrm{Si}$ structure or similar metal-oxide-semiconductor (MOS) structures with native oxide. ${ }^{13,14}$ Among them, the obstacle of the indirect-band gap nature of $\mathrm{Si}$ was never circumvented, so no emission corresponding to band gap energy occurred. In this article, we report that MOS structures on Si with the oxide grown by well-controlled rapid thermal oxidation (RTO) could have the emission corresponding to band gap energy of Si. The physical reason is discussed.

MOS structures that exhibit electroluminescence have very thin oxide grown by RTO at $900{ }^{\circ} \mathrm{C}$. The gas flows are $500 \mathrm{sccm}$ nitrogen and $500 \mathrm{sccm}$ oxygen at the pressure of 500 mbar. Two types of samples had been experimentally investigated. They were grown on $p$-type and $n$-type $\mathrm{Si}$ with the oxide of 2.3 and $3.3 \mathrm{~nm}$, respectively. The thickness was measured by ellipsometry and confirmed by x-ray reflectivity. The resistivity of the $100 \mathrm{~mm} p$-type and $n$-type (100) wafers used in this study is $1-5 \Omega \mathrm{cm}$ and $1-10 \Omega \mathrm{cm}$, respectively. Aluminum metal pads were then immediately deposited by evaporation on the oxide.

A typical continuous wave $(\mathrm{cw})$ current-voltage $(I-V)$ curve of the MOS on $p$-type $\mathrm{Si}$ without cooling is shown in Fig. 1, showing fair rectification characteristics. The voltage was applied between metal and Si. For the MOS on $p$-type $\mathrm{Si}$, significant current could flow through when the metal is negatively biased and vice versa for the MOS on $n$-type $\mathrm{Si}$.

\footnotetext{
a) Also at Graduate Institute of Electro-Optical Engineering. Electronic mail: cflin@cc.ee.ntu.edu.tw

b) Also at Graduate Institute of Electro-Optical Engineering.
}

Adapting the terminology of conventional diodes, we name this condition as forward bias and the opposite bias as reversed bias. The MOS on $n$-type $\mathrm{Si}$ has a similar $I-V$ curve except that its turn-on voltage is smaller because the Al Fermi level $\left(E_{f m}\right)$ is closer to the valence band of $\mathrm{Si}$ than the conduction band.

Under the forward bias, luminescence was clearly observed through an infrared viewer even with a current density as low as $0.67 \mathrm{~A} / \mathrm{cm}^{2}$. In the reversed bias, no luminescence was observed. The emission spectra were measured using a monochromator from the circular area of $2 \mathrm{~mm}$ diameter. Figures 2(a) and 2(b) show the measured spectra with the emission centered around 1150 and $1180 \mathrm{~nm}$ for the MOS on $p$-type and $n$-type $\mathrm{Si}$, respectively, under cw operation without cooling. Both correspond to the band gap energy $\mathrm{Eg}$ of $\mathrm{Si}$ slightly above the room temperature. The longer wavelength for the MOS on $n$-type $\mathrm{Si}$ is probably caused by the heating effect as a result of larger injection currents.

Different from other reports, ${ }^{13-15}$ no visible luminescence was measured from our devices. The previously reported visible luminescence from the MOS with native oxide $^{15,16}$ is probably due to luminescent centers or oxygen deficient defects in the native oxide. In our devices, because the oxide was grown by well-controlled RTO, it should have much less defect than the native oxide. Thus, visible luminescence is not observed.

Because the peak wavelength is longer than $1100 \mathrm{~nm}$, the output power was measured using an InGaAs detector. Figure 3 shows the measured $L-I$ curve for the MOS on $p$-type Si under cw operation without cooling. The curve shows a superlinear increase of the output power with the injection current. Because the Al pads are very thick, the light was observed to emit only from the periphery. If transparent electrodes were used, the output power should significantly increase.

The physical reason for the emission around Eg is discussed as follows. The discussion is based on the MOS on $p$-type Si only because the physical reason for the MOS on $n$-type $\mathrm{Si}$ is similar. For the MOS on $p$-type $\mathrm{Si}, E_{f m}$ increases 


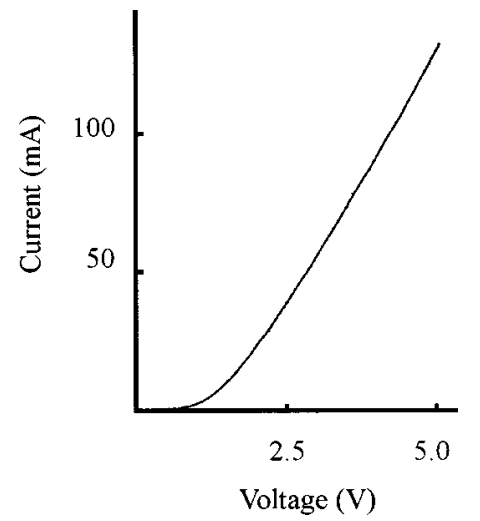

FIG. 1. The cw $I-V$ curve of the MOS structure on $p$-type silicon at room temperature.

with the forward bias. Electrons then tunnel from metal to $\mathrm{Si}$ and holes tunnel from Si to metal. Theoretical analysis had shown that the major contribution of current comes from the tunneling of electrons instead of holes for the MOS on p-type $\mathrm{Si}^{17}$ Therefore, as $E_{f m}$ moves up to the conduction band edge $(E c)$, the tunneling current increases significantly because the tunneling electrons could fill in many suddenly appeared states in the conduction band. The large current causes the applied voltage indicated in Figs. 1 and 2 to drop mostly across the resistance part in the substrate, so the voltage across the oxide is still less than the breakdown voltage of oxide. On the other hand, the negative bias of metal causes the band of the $p$-type silicon to bend up near the $\mathrm{Si} / \mathrm{SiO}_{2}$ interface, as schematically shown in Fig. 4, leading to the formation of a potential well for hole confinement. An accumulation layer of holes near the interface is then formed.

(a)

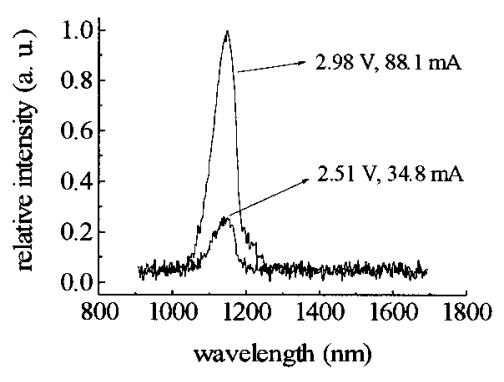

(b)

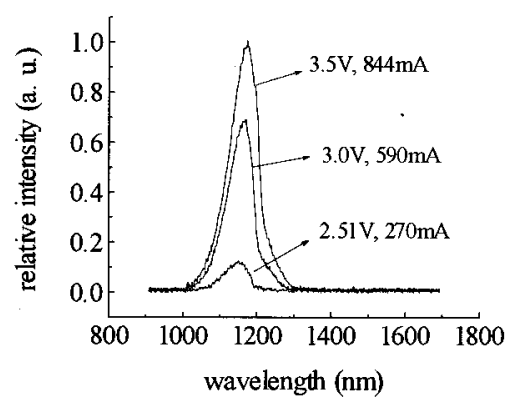

FIG. 2. The measured spectra under $\mathrm{cw}$ operation without cooling from the MOS on (a) $p$-type $\mathrm{Si}$ (b) $n$-type Si.

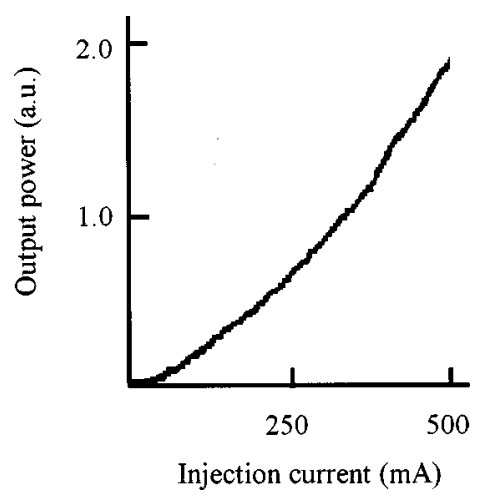

FIG. 3. The $L-I$ curve of the electroluminescence from the MOS on $p$-type $\mathrm{Si}$.

Consequently, huge population of the holes and the tunneling electrons simultaneously appear in the well region.

Although there are many electrons and holes in the same region, radiative recombination in the indirect-band gap material does not necessarily happen because the momentum still has to be conserved. However, the requirement of momentum conservation could be relaxed if some mechanisms are provided in the structure. The possible reasons for providing extra momentum to make radiative recombination happen are discussed in the following.

The hole in the accumulation region of the $p$-type semiconductor is confined in the $\mathrm{Si} / \mathrm{SiO}_{2}$ interface, and its wave function becomes localized. The localization of the hole creates the spread of lattice momentum in the reciprocal lattice due to the uncertainty principle. The momentum separation between electrons in the $\Delta$ valleys and the holes is then reduced to some extent, depending on the size of the hole wave function, as shown in Fig. 5. With the band filling effect, the spread of the wave function of holes in momentum space does not have to be as large as the separation of the conduction-band minimum and the valence-band maximum in Si crystal. On the other hand, even if the spread of lattice momentum is insufficient by itself to compensate for the momentum separation, it could make other mechanisms such as interface roughness, phonon, and impurity scattering more easily provide enough momentum for the radiative recombination. The hole confinement should be essential to have radiative recombination since the photoluminescence on the same sample is much weaker (not detectable in our setup) at

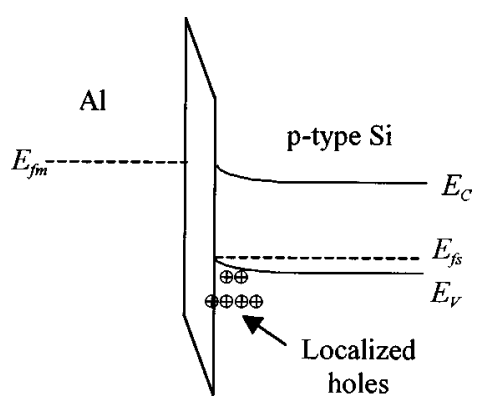

FIG. 4. A schematic of the band diagram with the metal of the MOS on $p$-type Si negatively biased. 

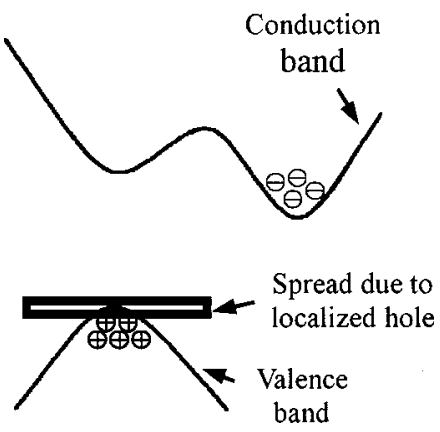

FIG. 5. A schematic of the spread of hole wave function in momentum space with respect to the momentum separation between electrons in the $\Delta$ valleys and the holes.

room temperature. The complete picture of the radiation process is not well known and is still under investigation.

The emission energy corresponding to 1150 and 1180 $\mathrm{nm}$ is slightly less than the Si band gap energy at the room temperature. The reasons could be threefold. First, the band gap shrinkage with the increasing carrier density ${ }^{18}$ due to the band gap renormalization effect might result in the redshift. Second, the interruption of Si periodical structure at the interface causes band bending and the band gap shrinkage. ${ }^{19}$ Third, the sample should be heated up under the large injection current, leading to the reduced band gap energy. The ohmic heat could also explain the MOS on $n$-type Si emitting light at a longer wavelength because a larger current is injected to it. On the other hand, the band-filling effect at a large injection current could cause the blueshift of the emission peak, ${ }^{18}$ but this effect is not sufficient to counter balance the above three factors.

In conclusion, electroluminescence corresponding to $\mathrm{Si}$ band gap energy from MOS structures on both $p$-type and $n$-type $\mathrm{Si}$ is observed. With very thin oxide grown by RTO, the MOS structures behave like light emitting diodes. Under forward bias, luminescence is observed even with a current density as low as $0.67 \mathrm{~A} / \mathrm{cm}^{2}$. The physical reason for the electroluminescence is attributed to the localized wave functions of holes, leading to the spread of momentum. The extra momentum required for the electron-hole radiative recombination is thus provided.

This work is supported in part by the National Science Council, Taipei, Taiwan, R.O.C. under Contract Nos. 882218-E-002-004, NSC88-2215-E-002-021, and NSC2112M-002-038.

${ }^{1}$ L. T. Canham, Appl. Phys. Lett. 57, 1046 (1990).

${ }^{2}$ N. Koshida and H. Koyama, Appl. Phys. Lett. 60, 347 (1992).

${ }^{3}$ S. Lazarouk, P. Jaguiro, S. Katsouba, G. Masini, S. La Monica, G. Maiello, and F. Ferrari, Appl. Phys. Lett. 68, 2108 (1996).

${ }^{4}$ S. Schuppler et al., Phys. Rev. B 52, 4910 (1995).

${ }^{5}$ D. B. Geohegan, A. A. Puretzky, G. Duscher, and S. J. Pennycook, Appl. Phys. Lett. 73, 438 (1998).

${ }^{6}$ L. S. Liao, X.-M. Bao, X.-Q. Zheng, N.-S. Li, and N.-B. Min, Appl. Phys. Lett. 68, 850 (1996).

${ }^{7}$ W. Skorupa, R. A. Yankov, I. E. Tyschenko, H. Frob, T. Bohme, and K. Leo, Appl. Phys. Lett. 68, 2410 (1996).

${ }^{8}$ G. Franzo, F. Priolo, S. Coffa, A. Polman, and A. Carnera, Appl. Phys. Lett. 64, 2235 (1994).

${ }^{9}$ M. Matsuoka and S. Tohno, Appl. Phys. Lett. 71, 96 (1997).

${ }^{10}$ D. R. Baigent, R. N. Marks, N. C. Greenham, R. H. Friend, S. C. Moratti, and A. B. Holmes, Appl. Phys. Lett. 65, 2636 (1994).

${ }^{11}$ R. Birkhahn and A. J. Steckl, Appl. Phys. Lett. 73, 1700 (1998).

${ }^{12}$ M. Garter, J. Scofield, R. Birkhahn, and A. J. Steckl, Appl. Phys. Lett. 74, 182 (1999).

${ }^{13}$ Y. Q. Wang, T. P. Zhao, J. Liu, and G. G. Qin, Appl. Phys. Lett. 74, 3815 (1999).

${ }^{14}$ J. Yuan and D. Haneman, J. Appl. Phys. 86, 2358 (1999).

${ }^{15}$ G. F. Bai, Y. Q. Wang, Z. C. Ma, W. H. Zong, and G. G. Qin, J. Phys.: Condens. Matter 10, L717 (1998)

${ }^{16}$ D. L. Griscom, J. Ceram. Soc. Jpn. 99, 899 (1991).

${ }^{17}$ C. W. Liu, M. H. Lee, C. F. Lin, I. C. Lin, W. T. Liu, and H. H. Lin, 1999 IEEE International Electron Devices Meeting, Washington, DC, December 1999.

${ }^{18}$ H. C. Casey, Jr. and M. B. Panish, Heterostructure Lasers, Part A (Academic, Orlando, FL, 1978).

${ }^{19}$ S. M. Sze, Physics of Semiconductor Devices (Wiley, New York, 1981). 